\title{
Neoantigen Cancer Vaccines: Real Opportunity or Another Illusion?
}

\author{
Karen Manoutcharian ${ }^{1}\left[\right.$. Jesus Guzman Valle ${ }^{1} \cdot$ Goar Gevorkian ${ }^{1}$
}

Received: 1 February 2021 / Accepted: 17 April 2021 / Published online: 28 April 2021

(c) L. Hirszfeld Institute of Immunology and Experimental Therapy, Wroclaw, Poland 2021

\begin{abstract}
In this communication, we will analyze some important factors and immunological phenomena related to neoantigen cancer vaccines, with particular emphasis on recently published Phase I clinical trials. Several obstacles and issues are addressed that challenge the current paradigm and inquire if neoantigens, which are essentially single-use vaccine candidates, are legitimate targets to induce protective immune responses with regard to the evolving mutational landscape. We also share insights into the striking similarities between cancer and antigenically variable pathogens and suggest that any successful vaccine against either should demonstrate a similar property: efficient induction of a diverse pool of immune cells equipped to prevent immune escape. Hence, to confront antigenic variability directly, we have employed our innovative vaccine concept, Variable Epitope Libraries, composed of large combinatorial libraries of heavily mutated epitopes, as a "universal" vaccine platform. Collectively, we offer critical analyses on key issues, which ultimately reflect on the prospective clinical relevance of personalized neoantigen vaccines which is still undefined.
\end{abstract}

Keywords Neoantigen cancer vaccine $\cdot$ Variable epitope library $\cdot$ Immune escape

\section{Rationale Behind Neoantigen Vaccines}

The consensus on the use of neoantigens (NeoAgs) as potential targets in cancer immunotherapy relies on the fact that NeoAgs are mutated forms of wild-type self-antigens, which theoretically avoid central tolerance, and thus, represent a feasible strategy for the successful generation of vaccines (Blass and Ott 2021). Renewed interest in cancer vaccines is also due to data obtained from immune checkpoint inhibitor (ICI) and adoptive T cell transfer (ACT) immunotherapies whose success has been attributed to the presence of $\mathrm{T}$ cells reactive to NeoAgs (Verdegaal and van der Burg 2017). The reduced adverse effects of ICI immunotherapy have allowed for more aggressive therapy in clinical trials, which will undoubtedly generate new combinations of conventional treatment with immunotherapy. It is worth mentioning that the increased use of ICIs in the clinic and postmarketing studies have revealed that less than $40 \%$ of cancer patients are eligible for treatment, and only around $10 \%$ respond to

Karen Manoutcharian

karman@unam.mx

1 Instituto de Investigaciones Biomédicas, Universidad Nacional Autónoma de México (UNAM), AP 70228, Ciudad Universitaria, 04510 Mexico, DF, Mexico it (Haslam et al. 2020). The reasoning behind the use of NeoAgs as cancer vaccine targets is based on the putative evasion of immune tolerance mechanisms by mutated wildtype self-antigens, an idea present in immunology textbooks. Furthermore, impressive developments in next-generation sequencing (NGS) techniques along with single-cell assays have served as important anchors to invigorate the field of NeoAg vaccines. These technological advances have provided great opportunities to generate personalized treatments by efficiently determining the antigenic landscape of tumors at a specific point in time. Thus, a recent comprehensive analysis of multiple metastases, including intratumoral heterogeneity studies, from melanoma patients showed that (i) despite ubiquitous sharing of clonal and subclonal NeoAgs among tumors, the $\mathrm{CD} 8^{+}$response is limited to a small $(<10 \%)$ subset of Ags; (ii) vaccination with NeoAgs promotes an increase in NeoAg breath and $\mathrm{T}$ cell clonotype diversity, suggesting ineffective $\mathrm{NeoAg}$ cross-presentation as the primary mechanism for immunological ignorance; (iii) vaccination is necessary in combination with ICI since most NeoAg-specific $\mathrm{T}$ cell clones are naïve and often their numbers are below detection limits (Linette et al. 2019). However, vaccine production timelines remain a major obstacle (vaccine turnaround can range from 2-3 months), and importantly, we cannot state that these technological 
advances have fostered a conceptually novel approach in vaccine development. Similarly, the additional components of vaccines such as adjuvants, immune modulators/stimulators, delivery platform or immunization protocols may enhance vaccine efficacy, but they will never compensate for the "correct" immunogen. The most important component of any vaccine, including a cancer vaccine, is the immunogen itself, which should induce de novo or reactivate pre-existing protective immune responses. Even though experimental and some clinical data have shown potential evidence associating the success of immunotherapy to the presence of NeoAgs, we believe that the rationale behind this generalization is questionable and recent evidence from vaccine trials is proving otherwise. Accordingly, we do not believe that the identification and direct targeting of NeoAgs represent a final common pathway for immunotherapy.

\section{Findings from Recent NeoAg Vaccine Clinical Trials}

The idea of NeoAg vaccines is not new (Finn and Rammensee 2018); whole-cell tumor-derived vaccines carrying the full collection of NeoAgs have failed in several clinical trials during the last decades. Results from initial Phase I clinical studies on personalized NeoAg vaccines (reviewed in Blass and Ott 2021) have made misleading claims of immunogenicity in both, melanoma and glioblastoma, where only a minority of selected NeoAgs (5-10\%) induced CD8 ${ }^{+}$ $\mathrm{T}$ cell activation regularly, after $2-3$ weeks of incubation with unphysiologically high concentrations of corresponding peptide epitopes. In this manner, the authors were able to report over $50 \%$ general immunogenicity by combining with $\mathrm{CD} 4^{+} \mathrm{T}$ cell proliferation data. Furthermore, it has been reported that mutated peptides could not evoke an isolated $\mathrm{CD}^{+} \mathrm{T}$ cell response and that hundreds of identified mutations were not presented as peptide: MHC complexes on the cell surface. Moreover, we should consider that DNA mutation-induced NeoAgs only represent a small portion of the modified tumor antigen landscape. Interestingly, in the most recent study yet, despite evidence of long-term persistence (almost 4 years) of NeoAg-specific $\mathrm{T}$ cell responses in melanoma patients, the majority experienced recurrence, leading the authors to conclude that "neoantigen vaccination alone may not be sufficient enough to generate lasting, clinically effective antitumor immunity" (Hu et al. 2021). Long-term efficacy results of this nature will provide clear evidence on the clinical relevance of NeoAg vaccines. Importantly, considering that activation of $\mathrm{CD}^{+}$effector cells is a minimal requirement for a cancer vaccine, no NeoAg-specific $\mathrm{CD} 8^{+}$ $\mathrm{T}$ cell responses were detected in newly vaccinated patients, which unfortunately was a consistent finding ( $\mathrm{Hu}$ et al. 2021). A recent clinical trial reported the use of personalized
mRNA vaccines carrying validated NeoAgs, driver gene mutations, as well as predicted neoepitopes to treat patients with metastatic gastrointestinal cancer. Although the vaccine was able to induce NeoAg-specific $\mathrm{T}$ cell responses, no objective clinical responses were observed in any of the patients in the trial (Cafri et al. 2020). Recently, data from a Phase Ib study to evaluate a personalized cancer vaccine (iNeST, developed by Roche's Genentech and BioNTech), in combination with an ICI in patients with locally advanced or metastatic solid tumors, were presented at the American Association for Cancer Research Annual Meeting 2020. Although NeoAg-specific T cell responses were observed in the peripheral blood of most patients (77\%), the objective response rate (ORR) was low (8\%): only nine out of 108 patients responded after treatment; furthermore, the ORR without ICI treatment was only $4 \%$. This kind of unfavorable data reported by Big Pharma demonstrates the outcome of tremendous financial and human resource investment in NeoAg vaccines, indicating that there are sound reasons to question the outlook of NeoAg vaccines. Similar evidence has also been found with the once heavily favored "universal vaccines", based on tumor-associated antigens (TAAs), where even the presence of anti-tumor immune responses in vaccinated patients was ultimately ineffective, consequently resulting in the termination of several recent clinical trials due to the lack of survival benefits.

\section{General Deficiencies in Natural and Induced Anticancer Immune Responses}

To understand why NeoAgs are not strong vaccine immunogens, we should consider several drawbacks of this vaccine concept: first, NeoAgs have already been positively selected in the presence of immune pressure, and second, the permanently evolving mutational landscape generates NeoAgs that are not, and will not, be targeted by any current vaccine approaches (Marty et al. 2017). With regard to the former, many authors give merit to the (short-sighted) success of personalized vaccines in activating naïve $\mathrm{T}$ cells against NeoAgs that would otherwise not induce natural immune responses: the majority of NeoAgs will not induce natural responses due to their low immunogenicity (Linette et al. 2019). In the case of the tumor antigen landscape, immune responses, either natural or vaccine-induced, will lag with respect to the mutational evolution of NeoAgs. In other words, cellular responses will perpetually remain "behind in the race", despite their adaptability, specificity and memory, in their effort to eliminate heterogeneous cancer cells. While with traditional vaccines we could state that durable immunity is a hallmark of a successful vaccination, this might not be the case in cancer, given that the persistence of 
NeoAg-specific $\mathrm{T}$ cells perhaps indicates that these are not efficiently eliminating tumor cells. To this effect, it has been seen that NeoAg-specific T cells can still be found in matched, post-treatment tumor samples, in spite of NeoAg target loss (Verdegaal and van der Burg 2017). The outcome of anti-cancer immune responses depends largely on the complex interplay between $T$ cells and rapidly evolving epitopes; therefore, the speed and timing of the appearance of NeoAgs and responding T cell clonotypes are certainly undeniable factors. Only until recently has the issue of timing in the cancer mutational landscape been directly addressed and analyzed systematically. Accumulating data from clinical studies on ACT and ICI immunotherapies clearly show that the cancer immunopeptidome is permanently changing, with gain/loss of NeoAgs within the tumors of treated patients in response to immune pressure; and that timing, a previously unappreciated factor, should be considered in the design of vaccines and clinical trials (Verdegaal and van der Burg 2017).

\section{Reflections on Reductionist Biology in NeoAg Cancer Vaccines}

The history of science has shown us that it would be nearly impossible to find one example where many research groups have coincided on a similar approach to a given phenomenon and arrived at the same resolution simultaneously. For over 5 years now, researchers have been trying to transform NeoAgs into effective personalized vaccines; however, such "low hanging fruit" should hardly be expected to generate successful cancer vaccines, due to the reductionist thinking behind $\mathrm{NeoAg}$ vaccines and the modest results obtained up to now. In addition to the caveats already detailed, the ideas spearheaded by Marc H. V. Van Regenmortel concerning the lack of correlation between antigenicity and immunogenicity, as well as the general deficiencies in rational vaccine design (van Regenmortel 2021), lead us to conclude that the technological advances facilitating NeoAg vaccine development do not compensate for the theoretical rather than demonstrable principles behind NeoAg-based cancer vaccines. A prevalent paradigm in HIV vaccine design has been structure-based reverse vaccinology: researchers believed that epitopes that bind to broadly neutralizing antibodies would also induce similar antibodies when used as immunogens. However, we should consider that while antigenicity is a physical-chemical property, immunogenicity is a complex multistep biological phenomenon with many unknowns, which partly explains why an effective HIV vaccine remains elusive (van Regenmortel 2020). Although the field of personalized vaccines is relatively young, similar events will surely transpire, and the rational approach behind NeoAg-based immunotherapies will be pursued further.

\section{Common Obstacles Between Antigenically Variable Pathogens and Cancer}

Successful life-saving vaccines developed during the 1950s targeted pathogens bearing low levels of genetic/antigenic variability, and were based on whole pathogen, or cell-based immunogens; however, current efforts to generate analogous vaccines against antigenically variable pathogens (AVPs) and cancer, during the last decades, have led to many disappointments (Kissick 2018; Servín-Blanco et al. 2016). The field of vaccines against AVPs and cancer faces similar challenges, even though the former is concerned with pathogen-derived non-self Ags. Synchronous studies used to elucidate the molecular compositions of both tumor cells and T cell receptor (TCR) repertoires, employing NGS techniques and systematic single-cell DNA/RNA analysis, have revealed striking similarities between cancer cells and AVPs (a prominent representative is HIV), in terms of interactions between evolving epitopes and the immune system (Kissick 2018; Servín-Blanco et al. 2016). Iterative cycles of mutation, immune pressure and selection during tumor development may possibly induce similar effects seen in chronic viral infections. If so, it would reveal common critical challenges towards the development of vaccines against AVPs and cancer. The state of balanced coexistence between the opposing forces of immune pressure and immune escape has been shown to inevitably lead to a favorable outcome for AVPs and cancer, which may explain why NeoAgs are not immunogenic, and even if they are, will not be protective.

\section{Original Antigenic Sin in AVPs and Cancer}

Data from infectious disease research has demonstrated that even minimal differences or overlap in amino acids between mutated and corresponding wild-type epitopes dramatically narrow the emerging TCR repertoire, rendering T cells dysfunctional, unresponsive and/or exhausted (Klenerman and Zinkernagel 1998). Original antigenic sin (OAS) is a welldocumented phenomenon, initially described in influenza as imprinting (memory induction) by the initial viral infection on the antibody response to subsequent vaccination or infection with a mutant virus (Monto et al. 2017; Vatti et al. 2017). Despite long debates, there is now convincing evidence that reduced vaccine effectiveness (estimated as 40-60\%) is related to OAS (Monto et al. 2017). This phenomenon was later described for cytotoxic $\mathrm{T}$ lymphocyte (CTL) responses, where mice primed with lymphocytic 
choriomeningitis virus WE strain (LCMV-WE) were not able to generate an adequate CTL response against a mutated LCMV-WE virus, bearing a variant CTL epitope during a subsequent infection. These effects led to immune escape and impaired clearance of mutant viruses (Klenerman and Zinkernagel 1998). One of the most alarming cases was caused by the Sanofi Pasteur Dengue vaccine, which sensitized some of the dengue-naïve recipients to severe life-threatening disease. This effect was caused by antibody-dependent enhancement, an OAS-related phenomenon (Shukla et al. 2020). The possible involvement of OAS, a strictly pathogen-related phenomenon, unappreciated in cancer immunology, might contribute to the final outcome of tumor-immune interactions. To elucidate our hypothesis that OAS may have a detrimental role in cancer evolution and immune escape (Domínguez-Romero et al. 2020), we have generated preliminary experimental data (currently still in process), which highly suggests the presence of this phenomenon in cancer.

\section{Dynamic Immunogens Against Cancer and Beyond: Variable Epitope Libraries}

Despite the acknowledgment, for decades now, of the continuous mutations in tumor cells and the acceptance of cancer as a genetic evolutionary disease, current vaccine concepts are not addressing these critical issues adequately. At this point, only out-of-box thinking can redirect scientific innovation away from the current paradigm and may propel it towards authentic and viable alternatives. As an alternative and qualitatively different approach, we have developed an innovative vaccine concept, Variable Epitope Libraries (VELs), which defies conventional vaccine development platforms and paradigms. VELs are designed to specifically target AVPs and cancer, they are composed of combinatorial libraries carrying thousands to millions of mutated versions of defined epitopes or multi-epitope regions. The artificial origin of VEL immunogens will reduce the "undesirable" downstream effects of immune-edition on VEL-induced T cells, which seems to be a normal response during tumor development/progression, or after infection with AVPs. Importantly, the simultaneous presentation of a large number of epitope variants by immunization with VEL vaccines may recall responses of the past, induce responses against antigens present during immunization, and induce $T$ cells capable of recognizing future mutated antigens, thus reducing the chances of immune escape: in this manner, VEL immunogens are in a class all their own. They are capable of inducing the largest possible repertoire of both $\mathrm{B}$ and $\mathrm{T}$ cells, and we demonstrated their ability to induce HIV-1 broadly neutralizing sera in mice (half of Tier 2 HIV-1 isolates were neutralized; Charles-Niño et al. 2011), and inhibit tumor growth and metastasis in an aggressive triple-negative breast cancer model (Domínguez-Romero et al. 2020; Servín-Blanco et al. 2018).

A relevant topic of great interest at this time is COVID19 , and it should be stated that according to recent reports, many highly neutralizing anti-SARS-CoV-2 monoclonal antibodies, human convalescent sera, and human sera from recipients of the BNT162b2 mRNA vaccine demonstrated diminished neutralization potency against some emerging variants, indicating that antigenic variability was not addressed adequately during vaccine design (Chen et al. 2021). Vaccine developers are quick to adapt to viral mutations and are prepared to modify licensed vaccines, generating their "revised versions"; however, this virus has shown a clear tendency toward rapid antigenic variation, and a panSARS-CoV-2 vaccine is warranted (Mascola et al. 2021). Although massive vaccination is the ideal setting, a potential danger exists in COVID-19 vaccines in the form of OAS. If its presence is determined during disease, this will inhibit the natural immune response against newly emerging mutant variants, leading to catastrophic outcomes, as mentioned previously with the Dengue vaccine. Therefore, immune responses in vaccinated individuals should be analyzed in detail to characterize the response against variant viruses. We believe that VEL-based COVID-19 vaccines are valid alternatives to existing vaccines (which still need to prove safety and efficacy) to protect from current and future virus strains. We have described our proposed vaccine candidates' design in an April 2020 study (Manoutcharian et al. 2020).

\section{Conclusion}

Despite the pessimism shared by us and other researchers (Finn and Rammensee 2018; Kissick 2018), NeoAg vaccines will undoubtedly reach large Phase III trials and lead us back where we started from, with greater detailed information on the personalized nature of tumor immunity. Few cancer types have been targeted thus far for NeoAg-based treatment; though, we could expect similar results from any one of the more than 200 cancer types if the indications for this immunotherapy modality are expanded. However, NeoAg-based vaccines have already made one significant "contribution" in the field of cancer research and therapy; they have dramatically reduced the number of clinical trials using TAAs, oncogenes and other non-mutated antigens. This will free up human and financial resources and redirect them towards the development of alternative vaccine strategies. Kissick stated years earlier that if NeoAgs are not capable of inducing/ expanding more significant numbers and more diverse repertoires of $\mathrm{T}$ cells with increased specificity for tumor Ags, than those obtained upon vaccination with TAAs, "it will certainly not be worth the significant extra effort required to 
generate these individualized vaccines" (Kissick 2018): we firmly believe that the validity of this opinion still holds true.

Funding This work was supported by PAPIIT-UNAM (IN205216) and CONACyT (N283036), Mexico, to K. Manoutcharian.

\section{Declarations}

Conflict of interest The authors state no conflict of interest.

\section{References}

Blass E, Ott PA (2021) Advances in the development of personalized neoantigen-based therapeutic cancer vaccines. Nat Rev Clin Oncol 18:215-229. https://doi.org/10.1038/s41571-020-00460-2

Cafri G, Gartner JJ, Zaks T et al (2020) mRNA vaccine-induced neoantigen-specific $\mathrm{T}$ cell immunity in patients with gastrointestinal cancer. J Clin Investig 130:5976-5988. https://doi.org/10.1172/ JCI134915

Charles-Niño C, Pedroza-Roldan C, Viveros M et al (2011) Variable epitope libraries: new vaccine immunogens capable of inducing broad human immunodeficiency virus type 1-neutralizing antibody response. Vaccine 29:5313-5321. https://doi.org/10.1016/j. vaccine.2011.05.007

Chen RE, Zhang X, Case JB et al (2021) Resistance of SARS-CoV-2 variants to neutralization by monoclonal and serum-derived polyclonal antibodies. Nat Med. https://doi.org/10.1038/ s41591-021-01294-w

Domínguez-Romero AN, Martínez-Cortés F, Munguía ME et al (2020) Generation of multiepitope cancer vaccines based on large combinatorial libraries of survivin-derived mutant epitopes. Immunology 161:123-138. https://doi.org/10.1111/imm.13233

Finn OJ, Rammensee HG (2018) Is it possible to develop cancer vaccines to neoantigens, what are the major challenges, and how can these be overcome? Neoantigens: nothing new in spite of the name. Cold Spring Harb Perspect Biol 10:a028829. https://doi. org/10.1101/cshperspect.a028829

Haslam A, Gill J, Prasad V (2020) Estimation of the percentage of US patients with cancer who are eligible for immune checkpoint inhibitor drugs. JAMA Netw Open 3:e200423. https://doi.org/10. 1001/jamanetworkopen.2020.0423

Hu Z, Leet DE, Allesøe RL et al (2021) Personal neoantigen vaccines induce persistent memory $\mathrm{T}$ cell responses and epitope spreading in patients with melanoma. Nat Med 27:513-525. https://doi.org/ 10.1038/s41591-020-01206-4

Kissick HT (2018) Is it possible to develop cancer vaccines to neoantigens, what are the major challenges, and how can these be overcome? Neoantigens as vaccine targets for cancer. Cold Spring Harb Perspect Biol 10:a033704. https://doi.org/10.1101/cshpe rspect.a033704
Klenerman P, Zinkernagel RM (1998) Original antigenic sin impairs cytotoxic T lymphocyte responses to viruses bearing variant epitopes. Nature 394:482-485. https://doi.org/10.1038/28860

Linette GP, Becker-Hapak M, Skidmore ZL et al (2019) Immunological ignorance is an enabling feature of the oligo-clonal $\mathrm{T}$ cell response to melanoma neoantigens. Proc Natl Acad Sci USA 116:2366223670. https://doi.org/10.1073/pnas.1906026116

Manoutcharian K, Valle JG, Odales J (2020) Variable epitope librarybased COVID-19 vaccine for current and future related epidemics. Open J Pharmacol Pharmacother. https://doi.org/10.17352/ojpp. 000011

Marty R, Kaabinejadian S, Rossell D et al (2017) MHC-I genotype restricts the oncogenic mutational landscape. Cell 171:1272-1283. e15. https://doi.org/10.1016/j.cell.2017.09.050

Mascola JR, Graham BS, Fauci AS (2021) SARS-CoV-2 viral variantstackling a moving target. JAMA 325:1261-1262. https://doi.org/ 10.1001/jama.2021.2088

Monto AS, Malosh RE, Petrie JG et al (2017) The doctrine of original antigenic sin: separating good from evil. J Infect Dis 215:17821788. https://doi.org/10.1093/infdis/jix173

Servín-Blanco R, Zamora-Alvarado R, Gevorkian G et al (2016) Antigenic variability: obstacles on the road to vaccines against traditionally difficult targets. Hum Vaccine Immunother 12:2640 2648. https://doi.org/10.1080/21645515.2016.1191718

Servín-Blanco R, Chávaro-Ortiz RM, Zamora-Alvarado R et al (2018) Generation of cancer vaccine immunogens derived from major histocompatibility complex (MHC) class I molecules using variable epitope libraries. Immunol Lett 204:47-54. https://doi.org/ 10.1016/j.imlet.2018.10.008

Shukla R, Ramasamy V, Shanmugam RK et al (2020) Antibodydependent enhancement: a challenge for developing a safe dengue vaccine. Front Cell Infect Microbiol 10:572681. https://doi.org/ $10.3389 /$ fcimb.2020.572681

Van Regenmortel MHV (2020) Truth in science and in molecular recognition, post-truth in human affairs. J Mol Recognit 33:e2827. https://doi.org/10.1002/jmr.2827

Van Regenmortel MHV (2021) What does it mean to develop an HIV vaccine by rational design? Arch Virol 166:27-33. https://doi.org/ 10.1007/s00705-020-04884-0

Vatti A, Monsalve DM, Pacheco Y et al (2017) Original antigenic sin: a comprehensive review. J Autoimmun 83:12-21. https://doi.org/ 10.1016/j.jaut.2017.04.008

Verdegaal EME, van der Burg SH (2017) The potential and challenges of exploiting the vast but dynamic neoepitope landscape for immunotherapy. Front Immunol 8:1113. https://doi.org/10. 3389/fimmu.2017.01113

Publisher's Note Springer Nature remains neutral with regard to jurisdictional claims in published maps and institutional affiliations. 